\title{
A NEW VENTILATOR DESIGNED TO MEET THE PRESENT-DAY NEEDS OF MECHANICAL AID TO VENTILATION IN THE TREATMENT OF RESPIRATORY FAILURE*
}

J. Howland Auchincloss, JR., M.D.

MECHANICAL AID to ventilation can be applied in two general forms: ventilatory assistance and ventilatory control. ${ }^{1}$ Although pure ventilatory assistance requires apparatus not available commercially, ${ }^{2}$ the use of intermittent positive pressure breathing (IPPB, patient-triggered, pressure-limited) apparatus without automatic cycling and the more recently developed patient-triggered external respirator, ${ }^{3}$ also if used without automatic cycling, can best be classified under this general heading. Ventilatory control can be achieved in relatively pure form with the use of a fixed-cycle piston pump with leak-free connections to the patient; the Engström respirator ${ }^{4}$ embodies these concepts.

The designer of a ventilator may attempt to increase the potential usefulness of his machine by making it capable of function either as an assistor or as a controller. The importance of this effort does not lie principally in reducing the number of types of equipment which a hospital should have available for different types of patients. Its real value lies in the possibility, even a probability, of a change occurring in the status of a patient from a problem best treated with assistance to one requiring controlled ventilation or vice versa. This happens when a tachypnoeic patient is first connected to a ventilator. Initially a sensitive device is required to respond to the patient's efforts; at a later time, a device which ensures a minimum number of breaths per minute may be necessary. Exactly the reverse may occur as the patient recovers and demands the sudden changes of ventilation characteristic of the conscious state.

The principle of guaranteed minimum respiratory frequency represents such an effort to introduce flexibility and has been employed extensively in the United States. It was introduced by the manufacturers when it became apparent that the pressure-limited ventilator was being used in situations where more was required than assistance of the patients' own efforts. It is in effect whenever an IPPB machine is attached to a patient and regulated so that the automatic cycling system is functioning and yet the machine is still capable of being triggered by the patient or when the automatic cycling system is "standing by" and the patient is triggering the valve. When such a machine is applied to the patient, further regulation, at least in theory, involves mainly a selection of the recycling pressure and of the peak inspiratory flow rate. The widespread popularity of equipment embodying these features can be partly explained by this incorporation of flexibility into the determination of the respiratory frequency.

\footnotetext{
From the Department of Medicine, State University of New York Upstate Medical Center, Syracuse, New York. This study was supported in part by U.S. Public Health Service Grant PN-2014 and by a grant from the Hendricks Fund.
} 
A decided disadvantage of ventilatory assistance in its pure form or with pressure-limited apparatus is the instability of the ventilation actually achieved in the patient. This problem was first noted when mechanical aid to ventilation was employed during anaesthesia, ${ }^{5-8}$ but its occurrence during all forms of mechanical aid to ventilation may be taken for granted at a time when a majority of patients have disease of the thorax or its contents. It becomes extremely conspicuous when measurements of ventilation are made continuously. ${ }^{\circ}$ It is primarily a disadvantage because the change of ventilation is usually caused by a change in the condition of the patient's airway, lungs, or thorax, because the change is usually downward and leads to hypoventilation with hypercapnia and hypoxaemia, and because the change may not be easily reversed unless quickly detected and treated. One of the most serious examples of this problem is the accumulation of secretions beyond reach of the suction catheter with the subsequent development of hypoventilation, atelectasis, and secondary infection. As a result of this type of difficulty there has been an increasing interest in the type of ventilator which delivers a constant volume on each cycle.

It is possible to devise a machine which delivers through a bellows a predetermined amount of air to the patient on each stroke and in which the frequency of strokes is governed by the principle, discussed above, of guaranteed minimum respiratory frequency. This combination of features is more commonly found in machines designed for use in anaesthesia than in those intended by the manufacturer for use outside of the operating room. When such a machine is to be used with an open or non-rebreathing circuit, a problem arises as to the design of the intake (that portion of the machine at which inspired air enters for pressurization) and the control of the predetermined volume. The intake can be constructed so as to be open to the atmosphere at some point; the volume of the bellows must then be limited within the housing of the ventilator by a tray or other device. This is the usual arrangement when such a machine is used in a non-rebreathing or "anaesthesia" circuit. Such a machine is commonly referred to as a volume-limited ventilator or one which delivers constant volume. Alternatively, the intake may be closed to the atmosphere and the internall limiting device removed. Under these conditions, a measured quantity of ventilation can be created by the use of compressed gases and flow meters with nebulizers added to provide humidification. If the patient increases his respiratory frequency under such an arrangement, the tidal volume diminishes. Such a machine (at this point an idea rather than an accepted device) can properly be referred to as a ventilation-limited ventilator or one which delivers constant ventilation. From these considerations, it is apparent that the availability of a guaranteed minimum frequency has presented a choice not available to the designer of a machine powered by a fixed-cycle pump. A machine which can furmish either constant volume or constant ventilation depending on its settings can be said to have both a volume-limited mode and a ventilation-limited mode of operation. Since the physician rarely wishes to impose an arbitrary upper limit on the value of ventilation, there would not appear to be a great need for the ventilation-limited mode. However, in patients with profound hypercapnia it is often desirable to increase the ventilation in a slow and deliberate fashion, and a direct way in 
which to accomplish this objective is to design the ventilator so as to make ventilation a controlled quantity. The designer, at least, cannot exclude a priori the possibility that a ventilation-limited mode has usefulness, and it should be furnished as an option.

A deficiency of almost all ventilators used in open circuits is the absence of automatic monitoring systems for immediate recognition of leakage. Leakage can indeed by identified indirectly by use of pressure allarm systems, as in the case of the Claude Bernard respirator and the respirator of Van Bergen ${ }^{10,11}$ or it can be identified directly but discontinuously by manual operation of a spirometer, as in the case of the Engström respirator. ${ }^{4}$ To date, the author knows of no machine which has a leak-detection system employing volumetric principles integrated into its design and functioning at all times. Prompt detection of leakage is particularly important when volume-limited or ventilation-limited machines are used, since these devices cannot compensate for that portion of the inspiration lost in the leak.

The simplest problems in the mechanical design of such a ventilator are those around which much discussion has occurred in the past. There can be no argument today that some ability to vary the inspiratory flow rate and thus to control the duration of inspiration, protection of the patient against excessive pressure as a secondary, limiting factor, unrestricted selection of air-oxygen ratios, and achievement of full saturation of the inspired air with water vapour are all necessary. I believe that most physicians who frequently administer mechanical aid to ventilation encounter patients, at least occasionally, in whom it is desirable to observe the effect of and, perhaps, to use an added, completely optional negative phase. Controversy on this issue has persisted for so long that the question of whether or not the negative phase should be incorporated into ventilators as an option seems insoluble by the methods of formal research and must be solved by the accumulation of broadly based experience.

These remarks might be summarized by saying that further refinements in the design of ventilators should be concerned not so much with the problem of the pressure pulse but with the problem of reconciling solutions to problems of a diverse population of patients who must be cared for wherever they present themselves and who may change without notice from one treatment category to another. The problem of pulsing air into the trachea has long been solved in the mechanical sense. The contemporary problem of adapting this pulse to the control system of the patient while at the same time contesting the progression of thoracic disease and guaranteeing the patient a minimum level of ventilation still justifies further efforts in design. The further problem of eliminating the element of human skill from the detection of leaks, so necessary in hospitals where personnel changes frequently, has also not been solved on a broad scale. An attempt to solve all of these problems of necessity results in the creation of a new design, actually a design of a circuit rather than one of a pump. Since a circuit can be constructed without the aid of a pneumatic engineer, the solution can be attempted with a minimum of special equipment.

The ventilator to be presented can be described as a constant-volume or constant-ventilation ventilator with patient triggering, guaranteed minimum rate, 
automatic measurement of expired air tidal volume on a breath-to-breath basis, and optional negative phase when used on the constant-ventilation mode. Since it is designed for use in patients with ventilatory failure, it has an open or nonrebreathing circuit. It is entirely mechanical and pneumatic in operation except for the heaters in the nebulizers and the suction pump providing energy for the negative phase. It can be constructed out of commercially available components, with the exception of a few parts which must be specially fabricated. Use of the machine has demonstrated that it performs the functions which it was designed to accomplish.

\section{Design and Construction}

Power Unit (IPPB, Fig. 1)

This is primarily a commercially available, pressure-limited ventilator (minus external connections) with patient triggering, optional guaranteed minimum rate, inspiratory nebulization, and optional negative phase. It was found to be more economical to buy a pleural suction pump for the actual development of negative pressure than to use the venturi provided by the manufacturer. Whether or not this is done, it is still necessary in order to avoid premature cycling to have a feedback from the exhalation manifold of the power circuit to the power unit ("Negative phase feedback," Fig. 1), and therefore to use a pressure-limited ventilator of the type which has optional negative phase if a negative phase is incorporated into the machine. The nebulization should be inspiratory only and not continuous, because if this outlet is piped into the power circuit, as shown in Fig. 1, it gives the operator considerable control over the inspiratory flow rate. It is therefore labelled "Insp. Flow Rate C" in Figure 1. I have not found it necessary to add a flow-rate limiting device to the power unit, probably because the bellows and valves to be described act in this capacity. Indeed, the problem is to maintain as high an inspiratory flow rate as desired in some patients, and the nebulization control, as renamed in Figure 1, has been useful in the capacity.

The power unit is usually operated at $40 \mathrm{~cm}$. peak pressure, rarely higher. Such a high pressure is essential to the function of the ventilator as a constantventilation or constant-volume machine. No internal modifications in the unit used were necessary to obtain $40 \mathrm{~cm}$., but it was modified to be capable of reaching $60 \mathrm{~cm}$. in case the need for this pressure should arise. No other modifications were made, and it is therefore reasonable to state that the power unit could be used effectively as supplied by the manufacturer.

\section{Power Circuit}

This consists of a plexiglass box $f$ enclosing two bellows and connected to the outlet of the power unit. During inspiration air enters the box from the power unit because the expiratory manifold, EM2, is closed as a result of pressurization of its rubber diaphragm. Both bellows are then evacuated, the box pressure rises to its recycling pressure $(40 \mathrm{~cm}$. water) and then falls to atmospheric

Bennett model PR-IN or PR-2 + Emerson Respirator Company, Cambridge, Mass.

fAqua Instruments, Syracuse, New York. 


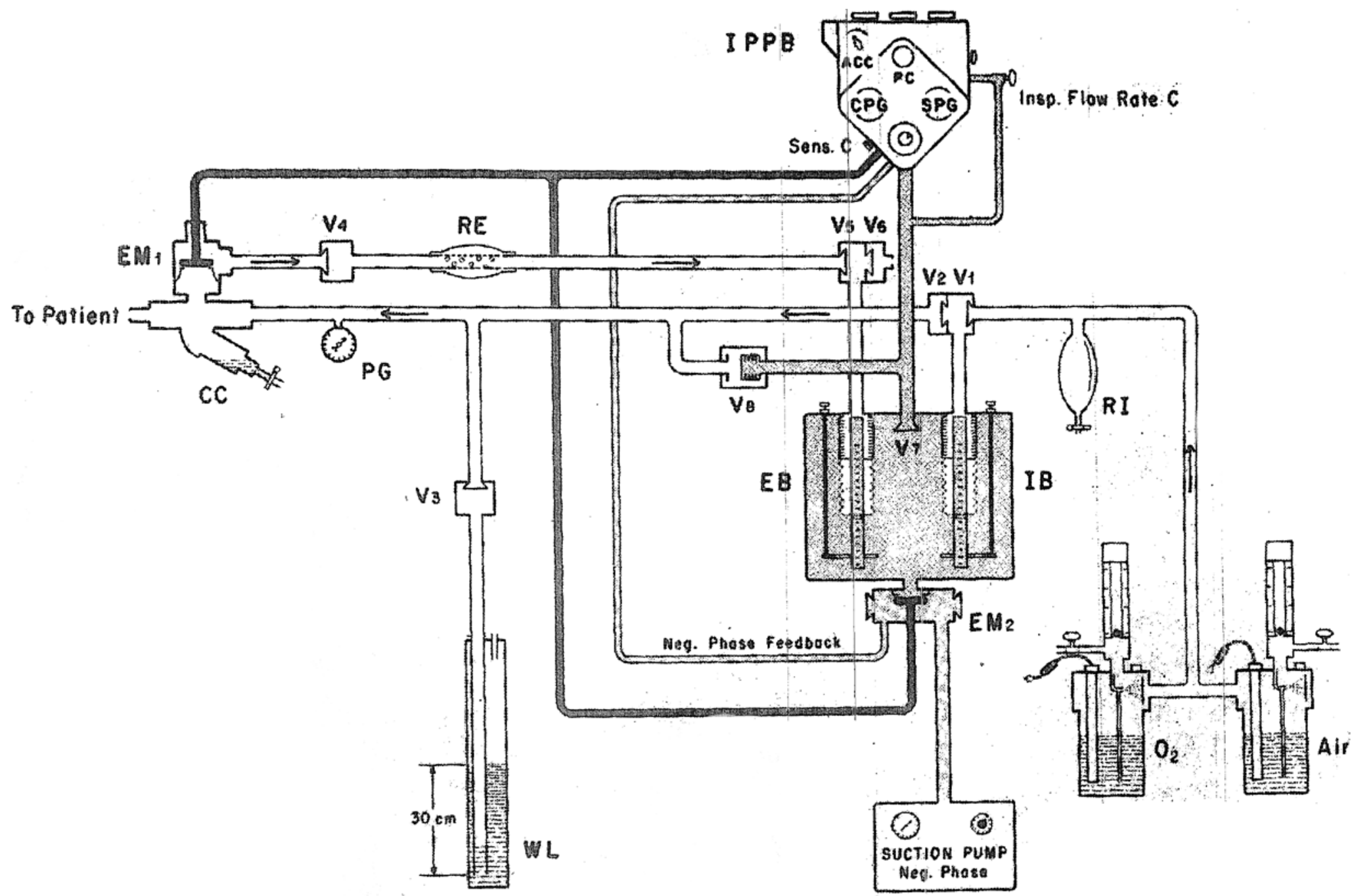

FigurE 1. Circuit diagram.

Power unit $(I P P B) . A C C=$ automatic cycling control for setting minimum respiratory frequency. $\mathrm{PC}=$ control determining the peak pressure applied to the bellows. CPG = gauge indicating instantaneous pressure applied to bellows (usually $0-40 \mathrm{~cm}$. water, occasionafly negative during expiration). $\mathrm{SPG}=$ gauge used in setting maximum pressure applied to bellows, usually $40 \mathrm{~cm}$. water. Sens. $\mathrm{C}=$ sensitivity control. Insp. flow rate $\mathrm{C}=$ control designed by manufacturer to furnish intermittent How to nebulizer, here used to accelerate the inspiratory flow rate.

Power circuit (shaded). Air in this portion of the apparatus cannot reach the patient and is used only for creation of positive or negative pressure about the bellows. V7 prevents reflux of air from the box when the small bellows of V8, the "manometric shunt," expand in response to an inspiratory effort of the patient.

Patient circult (unshaded). " $\mathrm{O}_{2}$ " and "Air" designate nebulizer-flow meter assemblies. $\mathrm{RI}=2 \mathrm{~L}$. anaesthesia bag used as inspiratory reservoir. WL $=$ water lock or safety valve. $\mathrm{PG}=$ gauge for estimating pressure applied to the airway of the patient. $C C=$ collector for water of condensation. $\mathbb{R E}=$ reservoir in expiratory circuit. $\mathrm{IB}$ and $\mathrm{EB}=$ inspiratory bellows and expiratary bellows, respectively.

Control cipcuit (black). Small-bore tubing connecting EM1 and EM2 to proper opening on power unit (IPPB).

levels. During exhalation both bellows descend by gravity (assuming normal operation) and displace air through $\mathbb{E M 2}$, whose diaphragm is no longer pressurized. The housing about $\mathbb{E M} 2$ with its valves is necessitated by the presence of the optional negative phase. The use of this arrangement plus the positivenegative ventilator and the suction pump renders the negative phase easily activated or inactivated. Unfortunately, it has not yet been found to be feasible to design an open circuit in which the negative phase can be used on the constant-volume mode. However, when the constant-ventilation mode is in use, the use of the negative phase is truly optional in that the operator, in order to 
introduce it, need only turn the switch and regulate the rheostat on the suction pump. Weighting of the bellows in order to achieve a negative phase is therefore not needed.

\section{Patient Circuit}

Inspired air is generated in the two flow meter-nebulizer systems labelled " $\mathrm{O}_{2}$ " and "Air" shown in the lower right-hand corner of Figure 1. The patient is assured of humidification; since heaters are employed, ${ }^{12}$ and the physician controls both the value of ventilation and the concentration of oxygen. The recent availability to us of an air compressor which is both quiet and of sufficient power makes it readily possible to employ constant ventilation with room air. Figure 1 shows the unit on the "constant-ventilation" mode. It can be converted to the "constant-volume" mode in a few seconds by (1) disconnecting one of the nebulizers from the circuit and thereby opening that portion of the circuit to the atmosphere; (2) setting the other nebulizer to furnish the desired gas mixture at an excessive flow rate, usually by employing the venturi in the $\mathrm{O}_{2}$ nebulizer which is furnished by the manufacturer for delivery of 40 per cent or 70 per cent oxygen; and (3) setting the volume-limiting tray beneath the inspiratory bellows (IB, Fig. 1) so as to deliver the chosen volume. The ventilator thereby furnishes minimum guaranteed ventilation rather than constant ventilation.

A 2 litre anaesthesia bag (RI, Fig. 1) serves as a storage area for inspired gas flowing from the nebulizers during the inspiratory time period when the constant-ventilation mode is in use. It fills during inspiration and empties through valve V1 into the bellows during expiration. The bellows fill from above downward by virtue of their own weight, so that air from the nebulizers cannot pass through V2 until the following inspiration. RI has no function during operation on the constant-volume mode, because that portion of the inspiratory circuit is then under atmospheric pressure throughout the entire respiratory cycle.

The rubber portion of the bellows employed is identical with that used in the Jefferson ventilator. The bottom of the bellows, however, has been fabricated as a removable disk, shaped like a watch glass, with a small plate valve (shown diagramatically in Fig. 1 but not labelled) in the centre placed so as to allow water of condensation to drain from the bellows. This valve in no way influences the performance of the ventilator and is of great importance during continuous operation on patients.

The inspiratory portion of the patient circuit contains also a connection with the "manometric shunt," V8 (to be discussed later), a water lock set usually at $30 \mathrm{~cm}$., a pressure gauge, and a side-arm for collection of condensate (CC, Fig. 1): This last feature can be omitted, and the photograph (Fig. 2) does not show it. In order to achieve simple, flexible connections to the patient, corrugated tubing and an exhalation manifold without a side-arm for a medication nebulizer are now employed, obviating the need for a supporting bracket. Removal of condensate at this point is a nursing responsibility, accomplished by separating and reattaching the connections, whereas elsewhere in the patient

"Air Shields, Hatboro, Pa. 


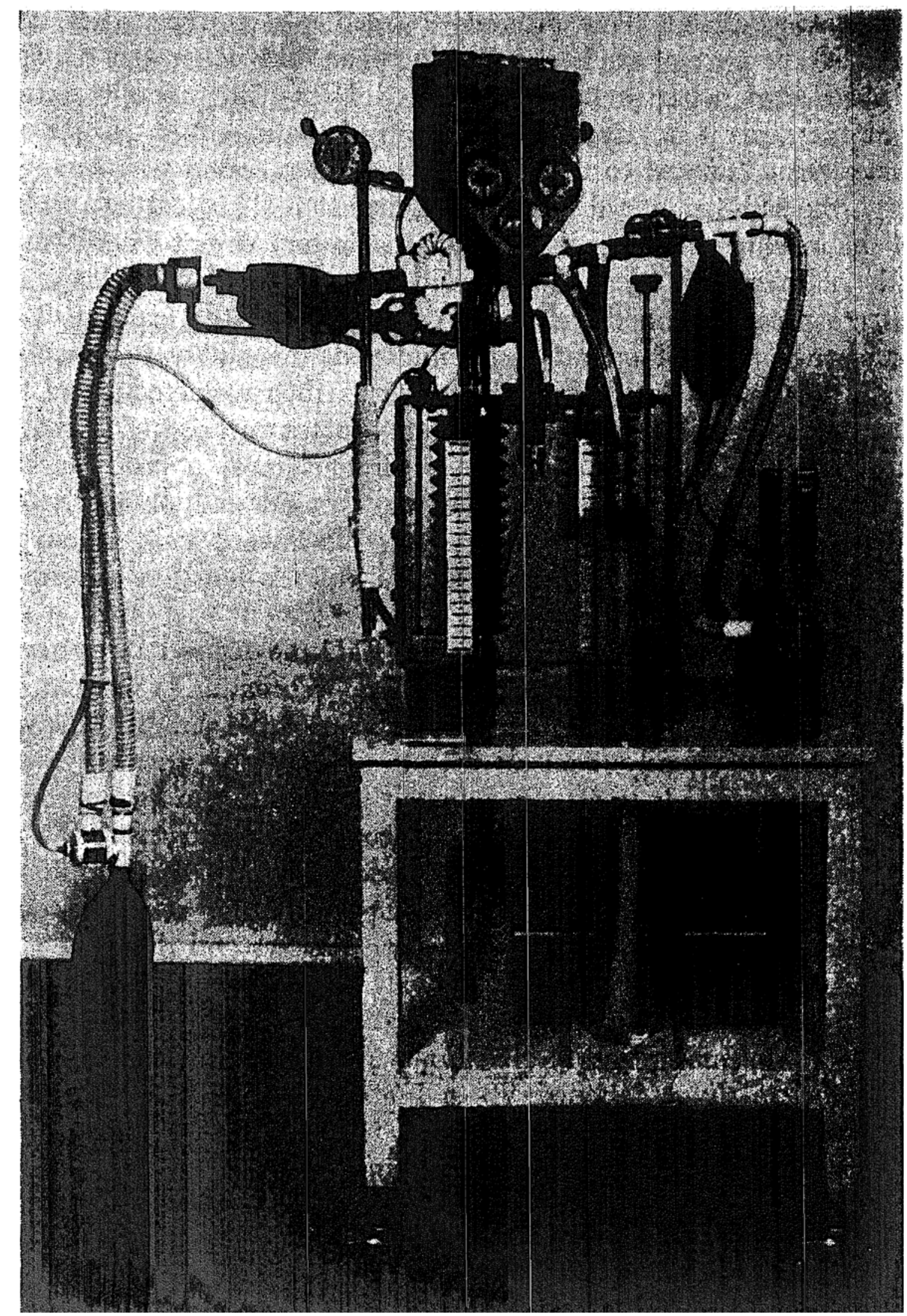

FIGURE 2. Frontal view of ventilator. High-pressure connections to power unit and nebulizers have been removed for illustrative purposes. Volumelimiting tray is in position under inspiratory bellows.

circuit it requires no manipulation. Connections are colour-coded in order to ensure proper reattachement.

Although EM1 is a standard collecting exhalation manifold, the monitoring action of this machine critically depends on a property of such a manifold which will go unrecognized in ordinary use: namely, its ability to pass air in exhalation around the unpressurized diaphragm only when there exists a positive pressure 
in the inspiratory circuit. This property is readily demonstrated if one sucks on the exhalation port of such a collecting manifold when the diaphragm is unpressurized. The negative pressure created by sucking merely expands the diaphragm and seals it tightly against the rim of the manifold. No air can pass. This is in extreme contrast to the very low resistance found when one breathes out through the same manifold in the usual direction. This feature results in the ability of the machine to detect leaks in the inspiratory circuit when the positiveonly mode of operation is employed, since the air leaking during inspiration cannot create pressure and therefore be represented in the expirate. When the positive-negative mode of operation is employed, the ability to detect leaks is theoretically lost, since the rubber diaphragm is then retracted away from the rim of the manifold as a result of the negative pressure existing at that time in the control circuit. However, under these circumstances a leak can in actual practice usually be recognized by observing a rapid and obviously excessive descent of the expiratory bellows as a result of an inward leak, e.g. air entering the patient circuit from the outside as a result of the negative pressure in the circuit.

Immediately after passing the diaphragm of EM1 the expired air passes through valve $\mathrm{V} 4$, which insures that there will be no rebreathing, since it prevents retrograde movement of air in the expiratory arm of the circuit. Thereafter the expiratory circuit is virtually identical with the inspiratory circuit. An expiratory reservoir, $\mathbb{R E}$, corresponds to $\mathrm{RI}$. Its function is to store the first portion of the expirate until the expiratory bellows can descend. By constructing the reservoir as shown in Figure 1, the resistance to its filling is so low that the first portion of expiration, whose velocity is relatively high, will not sweep through past valves V5 and V6 prior to the readiness of the bellows and therefore be lost to measurement. The patient circuit ends at valve V6.

The entire patient circuit including the bellows and bellows holders can be disassembled for cleaning and sterilization.

The manometric shunt, labelled V8 in Figure 1, is, in effect, a partial connection between the power circuit and the inspiratory portion of the patient circuit. It consists of a small bellows, approximately $2 \mathrm{~cm}$. in diameter, mounted in a valve housing, as shown in Figure 1. These bellows tend to assume a position of collapse during expiration because of their natural shape and because of the general absence of positive pressure in the power circuit tending to inflate them at this time. When the patient inhales, the slight negative pressure in the patient circuit tends to draw the bellows of $V 8$ into a position of inflation. A valve in the power circuit, $V 7$, prevents retrograde movement of air from the box; therefore, air must come from the power unit, which is thereby activated. When the power unit discharges, air cannot pass through the shunt because V8 is a bellows and not a true valve. It is a simple matter to show that this arrangement greatly increases the ability of the ventilator to respond to the inspiratory efforts of the patient.

Valves $\mathrm{V} 1$ and $\mathrm{V} 5$ are $\mathbb{J}$ valves in specially fabricated housings. $\uparrow$ The use of

- Warren E. Collins, Boston, Mass.

PAqua Instruments, Syracuse, N.Y. 
"J" valves is necessitated by the requirement of very low resistance in order to permit rapid bellows filling without recourse to the use of weight. "J" valves are also used on the housing of EM2, since resistance to airflow at this point must also be minimized. At other points small plate valves are used which can be inserted into the Tygon tubing out of which most of the patient circuit is constructed. Condensate drains through such valves, and with this type of installation their function can be verified by inspection.

The control circuit (black, Fig. 1) consists of small-bore tubing connecting the diaphragms of EM1 and EM2. It has the usual function of sealing these openings during inspiration.

\section{Perpormance of the Ventilator under Laboratory Conditions}

The ventilator was set to deliver a volume of $500 \mathrm{ml}$. at a frequency of 20 breaths per minute and thereafter connected in succession to three rigid containers whose volume was 50,80 , and 20 litres respectively. The compliance of these containers is equal to $0.05,0.08$, and 0.02 litres/cm. water. Similar

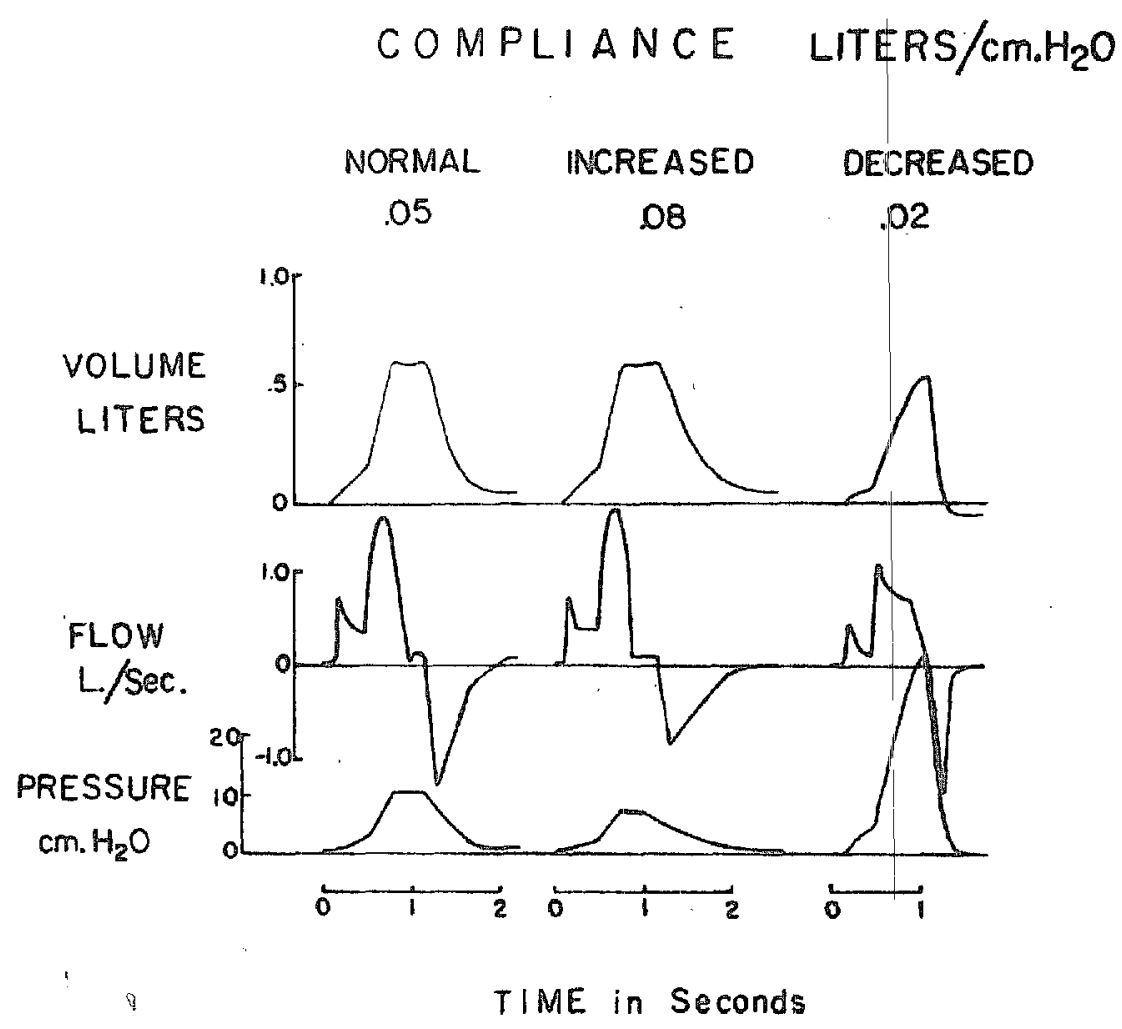

FIGURE 3. Tracings from oscillographic recordings showing pressure, flow, and volume relationships when ventilator, set on volume-limited mode, is connected to containers of $50 \mathrm{~L}$., $80 \mathrm{~L}$., and $20 \mathrm{~L}$. capacities. Pressure varies according to degree of elastic resistance.

determinations have been made by Elder et al. ${ }^{13}$ according to the method described by Elam et al. ${ }^{14}$ in order to compare the action of different ventilators. The results are shown in Figure 3. Tidal volume remains essentially constant despite variations in compliance, and, of equal importance, there is no prolongation of the inspiratory time period. Inspiration occupies between 0.3 and 0.4 of the respiratory cycle in each case.

- Courtesy Bennett Respiration Products, Santa Monica, Calif. 
In Figure 4 the machine is seen in use as a postive-negative ventilator which has been set to deliver constant ventilation. A standard "test lung" (rubber bag containing a foam rubber core) is used to simulate the patient. The response to the triggering impulse is indicated by the acceleration in respiratory frequency and the obligatory fall in tidal volume. In addition to demonstrating the contour of the expiratory negative pressure curve, the tracing demonstrates that patient triggering is possible on the negative phase.

\section{NEGATIVE PHASE with TEST / LUNG}

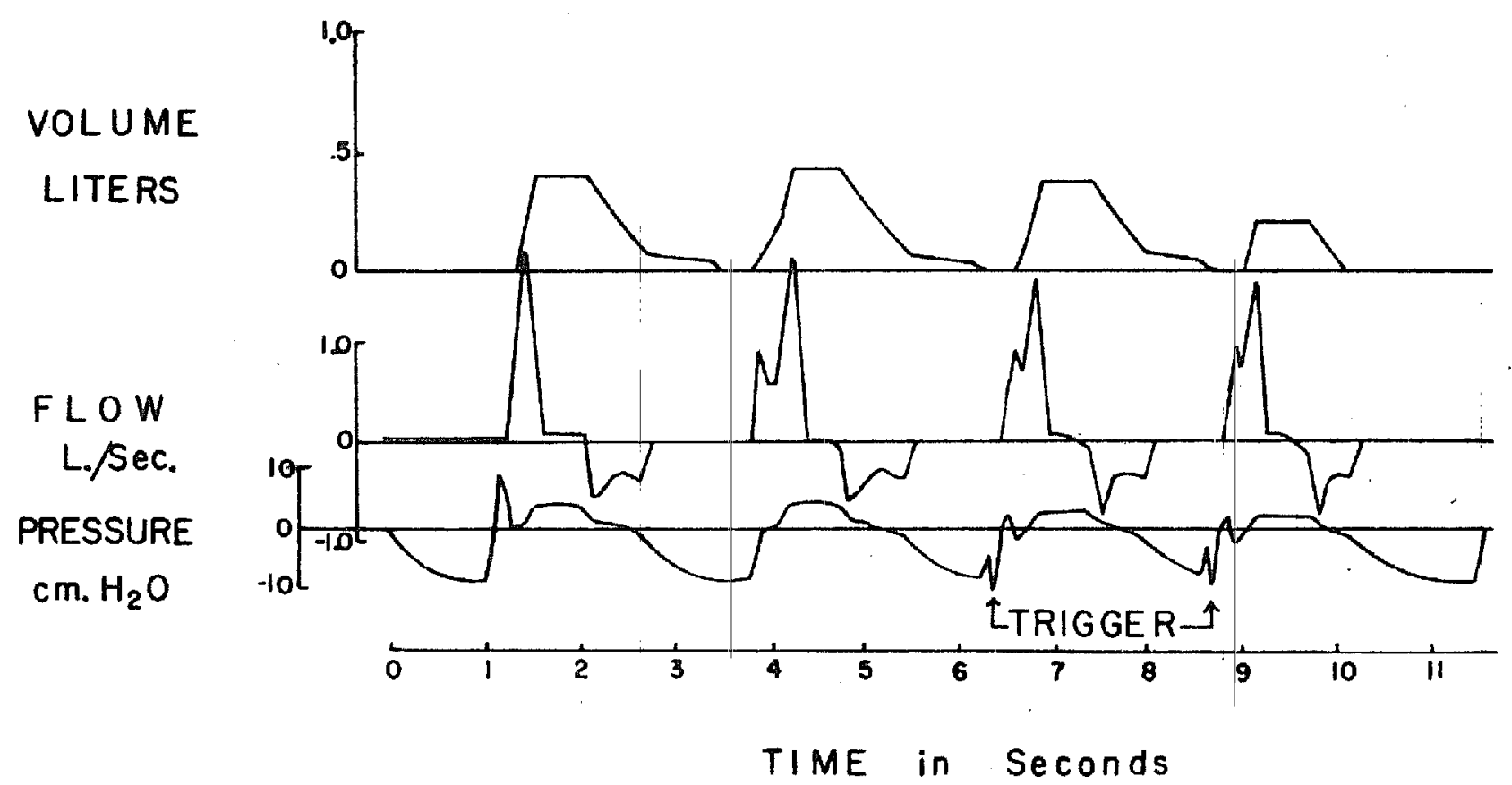

Figure 4. Pressure, flow, and volume relationships during operation of ventilator set on constant-ventilation mode with negative phase. The first two breaths are activated by machine; the last two are triggered by manual compression and decompression of a "test lung," e.g. a rubber bag with a foam rubber core.

Figures 5 and 6 demonstrate the response of the ventilator, now set as a positive-only ventilator, to triggering impulses when set on the constant-ventilation mode (Fig. 5) and the constant-volume mode (Fig. 6). The triggered breath is smaller than its predecessor with constant ventilation and equal in size to its predecessor with constant volume. The pressure registered at the mask during the triggering process is minus $4 \mathrm{~cm}$. of water. In separate determinations with a test lung, the triggering pressure was found to be slightly less than minus $1.5 \mathrm{~cm}$. of water, a figure which appeared to be quite acceptable in view of the fact that the power unit itself, when tested with the same strain gauge and recording system with only minimal external circuitry, required about minus $0.7 \mathrm{~cm}$. of water. According to this experiment the manometric shunt and inspiratory circuit added slightly less than $1 \mathrm{~cm}$. extra to the pressure required for triggering. Therefore, about $2.5 \mathrm{~cm}$. of the apparent triggering pressure of minus $4 \mathrm{~cm}$. of water obtained during the experiment on a normal subject displayed in Figures 5 and 6 can be explained by a relatively slow delivery of air in the initial phase of inspiration. The effect can also be seen in the 


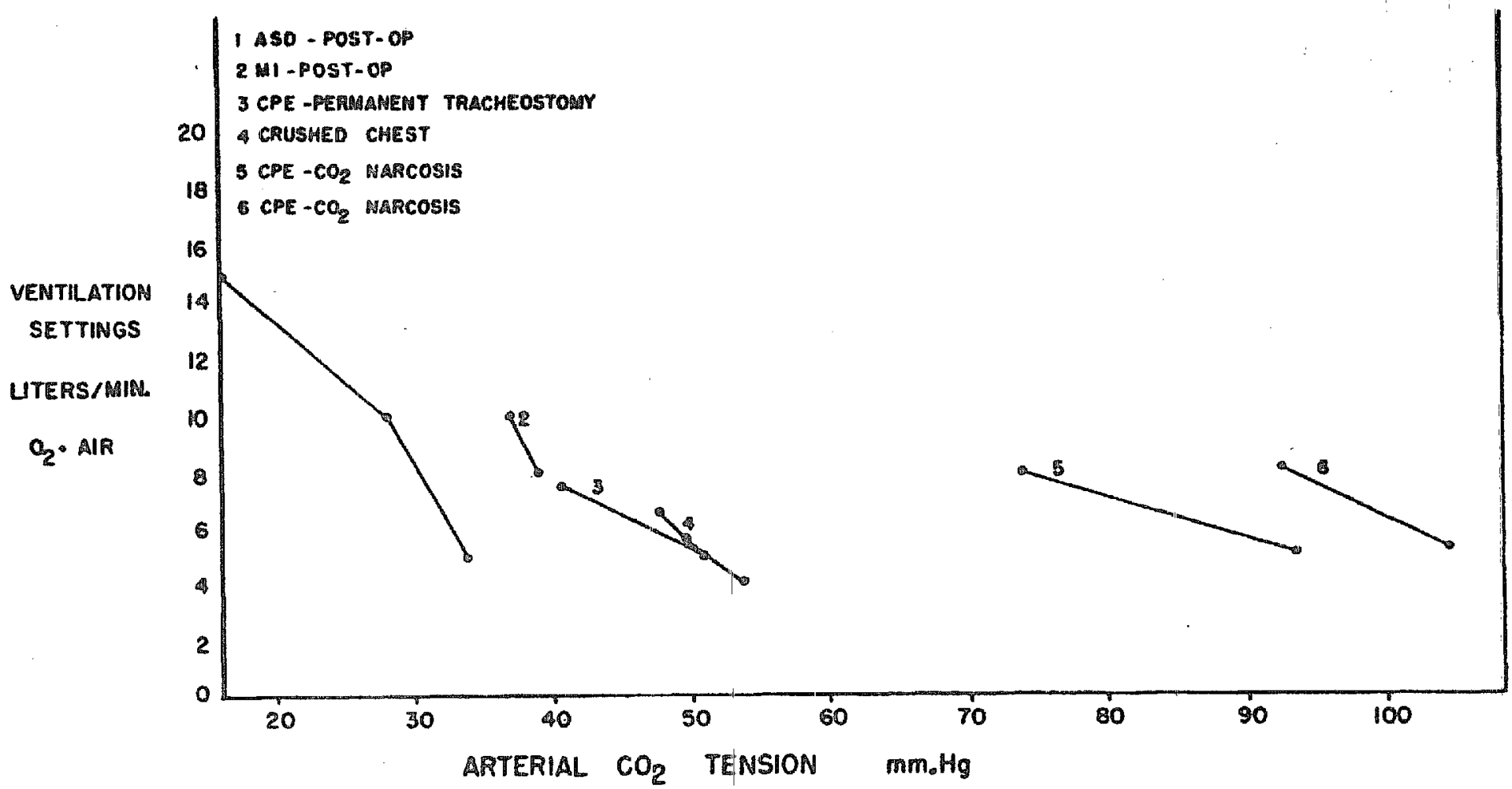

Frgune 7. Relationship between flow meter settings and $\mathrm{PaCO}_{2}$ values in six patients studied for short intervals while attached to the ventilator; the latter has been set on the constant-ventilation mode. $\mathrm{ASD}=$ atrial septal defect following opton repair. $\mathrm{MI}=$ mitral insufficiency. Patient was studied following valve replacement. $\mathrm{CPE}=$ chronic pulmonary emphysema.

therefore by no means a consistent finding. No effort was made to control the respiratory frequency except to maintain it at a minimum level of about 15 breaths per minute. The machine was used as a positive-only ventilator with 100 per cent oxygen or air-oxygen mixtures and was set on the constant-ventilation mode. Blood samples were drawn from indwelling arterial needles at times when no leaks were present as judged by equal descent of the inspiratory and expiratory bellows. Under these conditions it was logical to assume that the flow-meter settings were equal to the ventilation of the patient and that changing the ventilation should change the arterial carbon dioxide tension $\left(\mathrm{PaCO}_{2}\right)$. The downward slope of the lines connecting the points representing all six patients shows that such is the case. Thus the physician with such a ventilator has considerable control over the $\mathrm{PaCO}_{2}$ to the extent that he can change ventilation and eliminate leakage. By the same token it is obvious that considerable care must be taken in arriving at the proper settings. In this connection the values of Figure 7 should be regarded as trial settings only, since they were not used in these patients for sustained therapy.

The effectiveness of the negative phase is currently under investigation. Increases in cardiac output with its use have been observed in the two patients so far studied in whom circulatory depression existed, but there was no effect on cardiac output in a third patient who did not have circulatory depression.

In applying the ventilator to patients for the first time the author's current practice is to set the machine as a positive-only ventilator on the constant-ventilation mode at 5 or 10 litres per minute of 100 per cent oxygen and a minimum respiratory frequency of about 15 breaths per minute. This setting is instantly changed if it is felt to be incorrect. If the setting is too low, the respiratory 
frequency of the patient remains essentially unaltered, regardless of whether each inspiration triggers the machine, and the tracheal pressure gauge fails to register significantly positive values during inspiration. If, on the other hand, the ventilation imposed is excessive, the tracheal pressure rises rapidly to $30 \mathrm{~cm}$. of water and the ventilator pumps off the excess through the water lock. The proper setting of ventilation in the first hours of use of a constant-ventilation ventilator is a question to which no simple answer can be given. However, it should, in the author's opinion, involve some awareness of the level of ventilation prior to the application of the ventilator, regardless of whether this awareness is precisely quantitative or not. The application of a ventilation-limited, patienttriggered ventilator, set at a low or conservative value of ventilation with subsequent elevation of ventilation in the ensuing seconds or minutes to higher values if obviously indicated would seem to be a safe and effective way of approaching the problem. In this way the physician can begin to relieve the patient of the respiratory work load and to reduce the $\mathrm{PaCO}_{2}$ (if it is elevated) without suddenly imposing a drastic and dangerous change of ventilation. During this period of time he is only required to manipulate one control. When, with the aid of clinical observation, measurement of vital signs, and such laboratory information as is available, he decides that the correct ventilation has been achieved, he should have assurance that this ventilation will be maintained; and this assurance can only be furnished if the ventilator used is designed with this need in mind.

\section{Long-Term Experiences WiTh The USE OF THE VENTILAtOR ON PATIENTS}

The ventilator was used by the author as the ventilator of choice in six patients requiring mechanical aid to ventilation. Diagnosis, duration of use of the machine, and lowest $\mathrm{PaCO}_{2}$ value achieved during use of the machine are listed in Table I. In general the ventilator demonstrated the features previously discussed during this prolonged use. The leak detection feature was utilized frequently, both in detecting leaks in the connections leading to the patient and as an aid to determining the correct degree of inflation of the cuffs of tracheostomy and endotracheal tubes. The patient-triggering feature could be observed to be in use on numerous occasions. Malfunction of certain components occurred on various occasions, but in each instance the failure was detected, the machine was removed from the patient prior to any appreciable injury and was restored to use by replacement of the appropriate component. These problems have diminished with progressive refinement of the circuit, so that reliability in recent use has been satisfactory. Reliability is, no doubt, improved by the isolation of the cycling valve from the humidified air in the patient circuit so that moisture cannot enter the valve and cause sticking.

As part of the use of the ventilator on patients for prolonged periods of time an intensive effort was made to inform the special care nursing personnel of its action and purpose. Nurses were required to read the flow-meter settings, inspired volume, expired volume, and maximum tracheal pressure as well as to record the usual vital signs. A record of these readings at appropriate intervals 


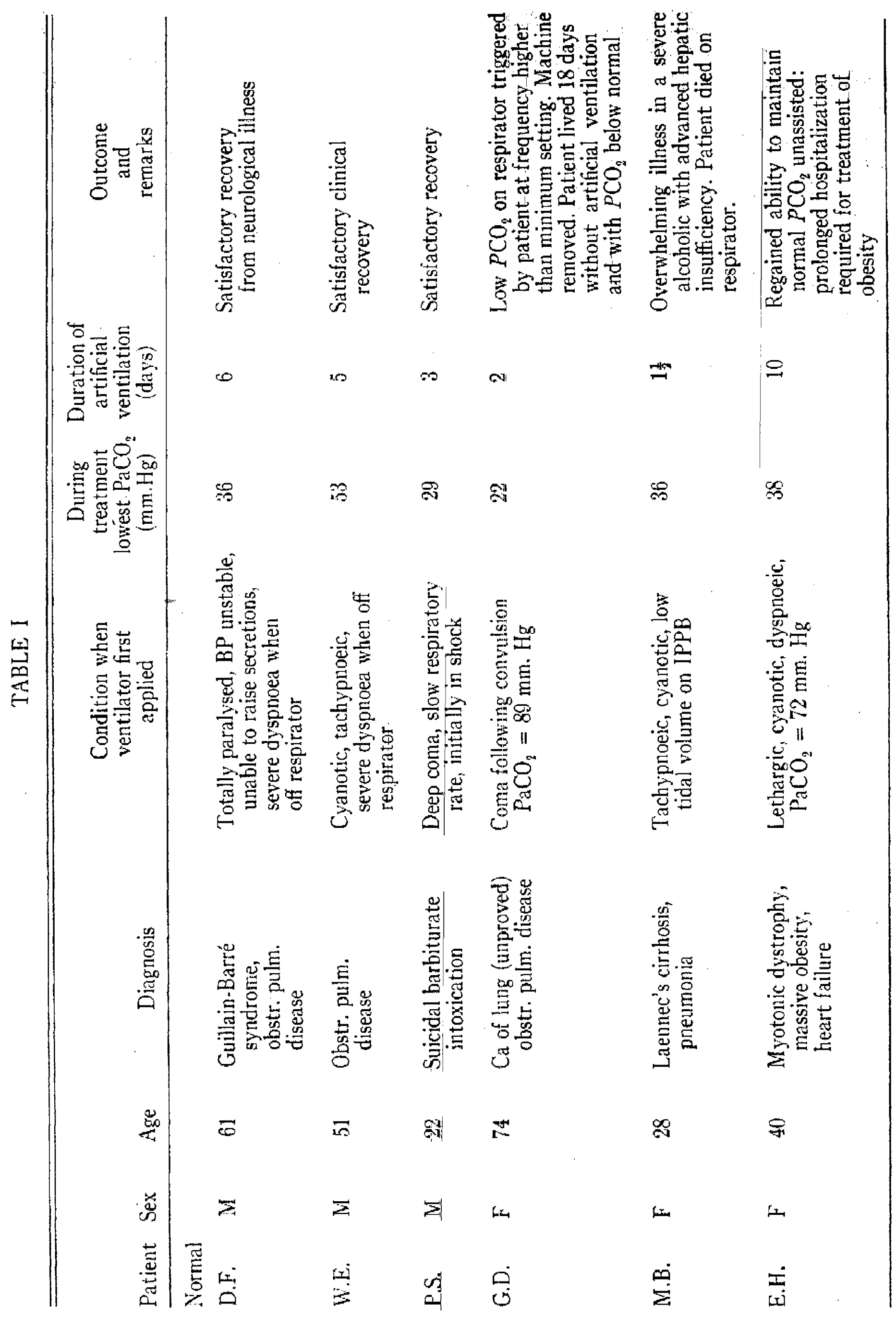


furnished evidence that the ventilation of the patient was maintained at given levels. Since no manipulation of controls was involyed in making these readings, change of nursing personnel presented minimal difficulty.

The ventilator was used as a constant-volume ventilator for all six of the patients after the desired or accepted level of ventilation was achieved. This practice was initiated prior to the availability of a good air compressor because it was well suited to the problem of easily available oxygen and unavailable compressed air. It meant that the patient received humidified 40 per cent oxygen at a minimum ventilation but could increase the ventilation to the extent that he or she could trigger the machine and thereby increase the number of powered inhalations each minute. In the event that the machine failed to respond to the patient's inspiratory effort (and no patient-triggered machine can resporid to every effort), it did not contest the effort, since the intake of the inspiratory circuit is open to atmosphere on such a setting. Every effort was made to increase progressively the tidal volume setting in accordance with the concept of developing adequate regional lung inflation. ${ }^{15}$ Tidal volumes of 1000 c.c. were achieved in some patients. It is the author's belief that such large tidal volumes given at slow respiratory frequencies may constitute an acceptable substitution for occasional deep breaths. Even with the availability of compressed air the use of 40 per cent oxygen, constant volume, and guaranteed minimum respiratory frequency represents settings applicable to many patients.

Measurement of unassisted ventilation or vital capacity was accomplished by disconnecting the inspiratory circuit between $\mathrm{V} 1$ and V2. This changed the ventilator into an unpowered open circuit with a patient-triggered, selfemptying spirometer $(\mathrm{EB})$ in the expiratory limb of the circuit. $\mathrm{EB}$ evacuates when the power unit is triggered by the manometric shunt or by manual activation of the cam on the cycling valve. This manoeuvre furnishes information about the patient's own ventilatory ability which has been found to be of great value in predicting the need for continued mechanical assistance to ventilation.

\section{DisCUSSION}

The features and performance of this ventilator have been presented, and it has been used sufficiently to demonstrate that these features, with the possible exception of the negative phase, are called into action quite frequently. The usefulness of the negative phase is under investigation, and in this study it is desirable to define its effects when ventilation is constant. Its non-availability when the constant-volume mode is in use represents a concession to simplicity and economy. Ideally, the negative phase should be available on both constantventilation and constant-volume modes; however, patients in whom the use of the negative phase is to be considèred are usually very ill and physically quiet and can be managed by the use of constant ventilation.

The number of features and the flexibility of this ventilator result in a device which is undeniably more complicated than other machines which might be said to perform the basic function of rhythmic inflation of the chest equally well. 
This creates problems in training and in maintenance. As regards training, complexities do not have to be fully understood by physicians and nurses involved with the care of the patient except in the case of the physician responsible for regulating the machine. For exannple, the author specifically instructs nurses to maintain and not to modify the settings on the power unit and flow meters. These settings are indicated by markings as well as by written order. If the nurse decides that the machine is functioning improperly and that the patient is not receiving the ventilation which was ordered, she is empowered to remove it from the patient and attach another ventilator and call a physician. A pressurelimited ventilator is kept on "standby" for such circumstances. The infrequency of such circumstances does not remove the necessity of preparation for their occurrence. The point of importance is that the number of rules relating to a ventilator must be kept to a minimum if changes in nursing personnel are to be dealt with easily, and it is highly desirable to have sufficient automation so that regulation of the machine is so infrequently necessary that it need not be a nursing responsibility. Failure of the ventilator should be identifiable independently of any change in the status of the patient. Detection of failure should not involve manipulations but should be possible by viewing its performance.

The extra components employed in this ventilator are, by and large, extremely reliable and are readily replaced when they become defective. The power unit is the only part of the machine which has required a specialized repair service and this is readily supplied by the manufacturer, since it is a standard item. Every effort has been made to avoid electronic control systems, both because of the explosion hazard and because of the difficulty of the repair problem which must result when such systems are used in community hospitals. It may then be said that this ventilator represents a compromise between simplicity and complexity, and that the compromise is mainly on the side of simplicity if it is compared with other devices which attempt to combine both therapeutic and monitoring functions.

\section{SUMMARY}

A description is presented of an internal ventilator which has the following features: (1) patient-triggering with guaranteed minimum frequency; (2) constant volume or constant ventilation as determined by the physician; (3) continuous, automatic monitoring of expired air tidal volume; (4) optional negative phase when ventilation is maintained at constant levels; (5) control over inspiratory flow rate and air-oxygen ratios. The ventilator has been tested under laboratory conditions and in separate series of short-term and long-term applications, and has been found useful because it provides the responsiveness and flexibility of patient-triggered apparatus, the basic security of constant ventilation or guaranteed minimum ventilation, and an automated system for monitoring ventilation. The rationale for design of a ventilator with these refinements is discussed and relates to the present population of patients requiring mechanical aid to ventilation. 


\section{RésUMís}

On présente la description d'un ventilateur interne qui a les caractéristiques suivantes:

(1) déclenchement par le malade avec un minimum garanti de fréquence;

(2) un volume constant ou une ventilation constante tel que prescrit par le médecin;

(3) un contrôle continuel, automatique du volume d'air courant expiré;

(4) une phase négative facultative quand la ventilation est maintenue à des niveaux constants;

(5) un contrôle sur le flot inspiratoire et sur les rapports air/oxygène.

Le ventilateur a été éprouvé en laboratoire et en séries d'application séparées à long et à court terme, et ill a été trouvé utile parce quill a la sensibilité et la flexibilité d'un appareil déclenché par le malade; il garantit la sécurité primordiale d'une ventilation constante ou d'une ventilation minimale, et il est muni d'un système automatique pour contrôler la ventilation.

La raison d'être d'un appareil possédant tous ces raffinements est démontrée, et l'on rappelle le nombre de malades qui peuvent bénéficier d'une aide mécanique à la ventilation.

\section{ACKNOWLEDGMENTS}

I wish to express my appreciation to Mr. Edward J. Toner and Mr. John L. Maines of the Physiology Instrument Shop, to Mr. Harold Garey and Michael Garey of Aqua Instruments, to Mr. H. L. Gage of Bennett Respiration Products, to Mr. Haven Emerson of the J. $\mathbb{H}$. Emerson Company, to the Nursing Service of the University Hospital, and to many other technicians, physicians, and nurses who have made this project possible.

\section{REFERENCES}

1. Auchincloss, J. H. Ir.; GilBert, R.; \& EpIFAno, L. Management of Acute Respiratory Failure in Patients with Chronic Respiratory Disease. New York State J. Med. 62: 3234 ( 1962).

2. Harries, J. R., \& Tyler, J. M. Mechanical Assistance to Respiration in Emphysema. Result with a Patient Controlled Servorespirator. Am. J. Med. 36: 68 (1964).

3. MARES, A.; Bocles, J.; \& MORGANT, L. A New Ventilatory Assister for Patients with Respiratory Acidosis. New Eng. J. Med. 268: 61 (1963).

4. Engström, C. G. Treatment of Severe Cases of Respiratory Paralysis by the Engström Universal Respirator. Brit. Med. J. 2: 666 (1954).

5. Bjonr, V. O., \& Engström, C. G. The Treatment of Ventilatory Insufficiency after Pulmonary Resection with Tracheostomy and Prolonged Artificial Ventilation. J. Thoracic Surg. 30: 356 (1955).

8. ELAM, J. O.; BROWN, E. S.; \& TENPAS, R. H. Carbon Dioxide Homeostasis during Anesthesia. I. Instrumentation. Anesthesiology 16: 876 (1955).

7. DoBmin, A. B., \& WYANT, G. M. Anaesthesia with Controlled Positive and Negative Pressure Respiration: Part II. Review of Cardiorespiratory Function and Acid-Base Homeostasis. Brit. J. Anaesth. 28: 353 (1956).

8. ETSTEN, B.; RETNoLDS; R. N.; \& RT, T. $\mathbb{H}$. Respiratory Effects of a Calibrated VolumeLimited Pressure-Variable Ventilator during Surgery. J. Appl. Physiol. 14: 736 (1959). 
9. Auchincloss, J. H. Jr., \& Keighlex, J. F. A Self-emptying Bellows Spirometer for Continuous Automatic Measurement of the Expired Air Tidal Volume of Patients Undergoing Artificial Respiration with Internal Pressure-Limited Respirators. Am. Rev. Resp. Dis. 88(2) : 257 (1963).

10. Mollanet, P., \& Pocidalo, J. J. Le Centre de Réanimation Respiratoire de l'Hôpital Claude-Bernard, Paris. Postgrad. Med. J. 37: 2 (1961).

11. Van Bergen, F. H.: Buckely, J. J.; Weatherhead, D. S. P.; Schultz E. A.; \& Gordon, J. R. A New Respirator. Anesthesiology 17: 708 (1956).

12. Wells, R. E. Jr.; Perera, R. D.; \& Kenney, J. M. Humidification of Oxygen during Inhalation Therapy. New Engl. J. Med. 268: 643 (1963).

13. Elder, J. D. Jr.; Duncalf, D.; Binder, L. S.; \& Harmel, M. H. An Evaluation of Mechanical Ventilating Devices. Anesthesiology 24: 95 (1963).

14. Elam, J. O.; KerR, J. H.; \& Janney, C. D. Performance of Ventilators: Effect of Changes in Lung-Thorax Compliance. Anesthesiology 19: 56 (1958).

15. Bendixen, H. H.; Hedley-WhIte, J.; ChIm, B.; \& Laver, M. B. Impaired Oxygenation in Surgical Patients during General Anesthesia with Controlled Ventilation. New Engl. J. Med. 269: 991 (1963). 